\title{
RAGE targeted strategies for Alzheimer's amyloid $\beta$ peptide induced blood brain barrier dysfunctions
}

\author{
Sholpan Askarova ${ }^{1 *}$, Andrey Tsoy', James C-M Lee ${ }^{2}$ \\ From Molecular Neurodegeneration: Basic biology and disease pathways \\ Cannes, France. 10-12 September 2013
}

\section{Background}

Blood-brain barrier (BBB) dysfunctions have been implicated in the development and progression of Alzheimer's disease. Cerebral endothelial cells (CECs) and astrocytes are the main cell components of the BBB. Although amyloid- $\beta$ oligomers have been reported to mediate oxidative damage to the CECs and astrocytes and trigger the downstream MAPK/ERK pathway, the cell surface binding site for $A \beta$ and exact sequence of these events have yet to be elucidated. In this study, the receptor for advanced glycation endproducts (RAGE) was postulated to function as a signal transducing cell surface receptor for $A \beta$ to induce ROS generation from NADPH oxidase and trigger downstream pathways for the phosphorylation of extracellular-signal-regulated kinases (ERK1/2) and cytosolic phosphorilase $\mathrm{A}_{2}\left(\mathrm{cPLA}_{2}\right)$.

\section{Materials and methods}

Immortalized CECs (bEnd3) and primary rat astrocytes were applied in this study. To confirm A $\beta$-RAGE binding, we examined the quantitative immunofluorescence microscopy (QIM) of cellular surface RAGE for primary astrocytes and bEnd 3 cells pretreated with oligomeric A $\beta 1-42$ at $+4^{\circ} \mathrm{C}$ (conditions in which the internalization of surface receptors is suppressed) and stained with antiRAGE primary antibody ( $\left.\mathrm{Ab}_{\mathrm{RAGE}}\right)$. To quantify the ROS production, we applied fluorescence microscopy of dihydroethidium (DHE) in the cells. Confocal immunofluorescence microscopy of double immunofluorescent labeled gp91-phox and $\mathrm{p} 47$-phox subunits was performed to characterize NADPH oxidase complex assembly. Western blot analysis was applied to characterize phosphorylation of cPLA2 $\alpha$.

${ }^{1}$ Center for Life Sciences, Nazarbayev University, Astana, Kazakhstan

Full list of author information is available at the end of the article

\section{Results}

We report that $A \beta_{42}$ competed with the anti-RAGE antibody ( $\left(\mathrm{b}_{\mathrm{RAGE}}\right.$ ) to bind to RAGE on the surfaces of CECs and primary astrocytes. In addition, $A b_{\text {RAGE }}$ abrogate $\mathrm{A} \beta_{42}$-induced ROS production and the co-localization between the cytosolic (p47-phox) and membrane (gp91-phox) subunits of NADPH oxidase in both cell types. $A b_{\text {RAGE }}$, as well as NADPH oxidase inhibitor and ROS scavenger suppressed $A \beta_{42}$-induced ERK1/2 and ${ }_{C P L A}$ phosphorylation in CECs. At the same time, only $A b_{\text {RAGE }}$, but not NADPH oxidase inhibitor or ROS scavenger, inhibited ERK1/2 and cPLA2 $\alpha$ phosphorylation in primary astrocytes.

\section{Conclusions}

This study demonstrated that $A \beta_{42}$ binding to RAGE on the $\mathrm{CEC}$ and astrocyte surfaces is required for NADPH oxidase complex assembling, resulting in subsequent ROS generation and phosphorylation of ERK1/2 and $\mathrm{CPLA}_{2}$ and suggested the presence of two different RAGE-dependent downstream pathways in CECs and astrocytes. Therefore, understanding precise molecular mechanisms underlying A $\beta$ mediated oxidative damage may provide new insights into the development of preventive and treatment strategies for AD.

\section{Authors' details}

${ }^{1}$ Center for Life Sciences, Nazarbayev University, Astana, Kazakhstan. ${ }^{2}$ Department of Biological Engineering, University of Missouri, Columbia, MO, USA.

Published: 13 September 2013

doi:10.1186/1750-1326-8-S1-P5

Cite this article as: Askarova et al:: RAGE targeted strategies for

Alzheimer's amyloid $\beta$ peptide induced blood brain

barrier dysfunctions. Molecular Neurodegeneration 2013 8(Suppl 1):P5. 